\title{
Coconstrucción de un modelo de desarrollo regional integral de la Universidad de Ibagué
}

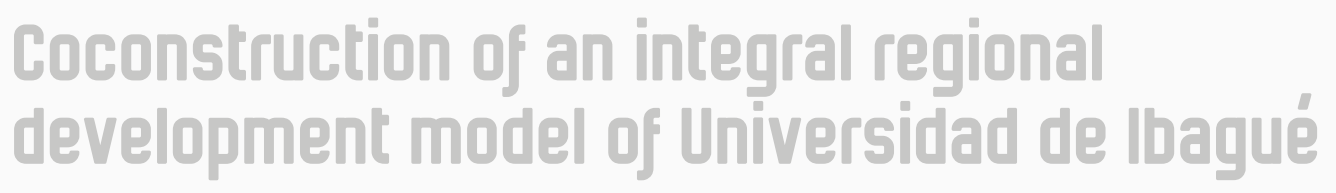

\author{
Lady Johanna Peñaloza Farfán ${ }^{1}$ \\ Hans Sanabria Gómez ${ }^{2}$
}

(2)

Recepción: 04/06/2020

Aprobación: 13/08/2020

Publicación: 18/12/2020

\section{Para citar este artículo:}

Peñaloza Farfán, L. J., \& Sanabria Gómez, H. (2020). Coconstrucción de un modelo de desarrollo regional integral de la Universidad de Ibagué. Indagare, (8), 126-136. https://doi.org/10.35707/indagare/810

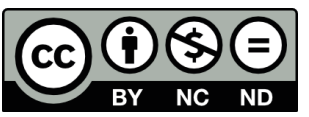

${ }^{1}$ Grupo de investigación Eulogos. Universidad de Ibagué. ORCID: 0000-0003-3920-7634. Correo electrónico: lady. penaloza@unibague.edu.co

2 Grupo de investigación EUlogos. Universidad de Ibagué. ORCID: 0000-0002-8665-5678. Correo electrónico: hans. sanabria@unibague.edu.co 


\section{Resumen}

Este proyecto parte de la misión que tiene la Universidad de Ibagué con el desarrollo regional, como estrategia para aportar a las dimensiones sociales en las que se encuentra inmersa. Se hipotetiza que la comunidad universitaria tiene diferentes percepciones del compromiso con el desarrollo regional y, además, que la elaboración del modelo preferiblemente debe ser integral y tener en cuenta las complejidades del contexto regional. Se propone como objetivo coconstruir un modelo de desarrollo regional integral de la Universidad de Ibagué a partir de la concepción que tienen los agentes, desde el enfoque cualitativo y la metodología basada en las teorías ancladas en contextos específicos de experiencia.

\section{Abstract}

This project starts from the mission that Universidad de Ibagué has to do with regional development, as a strategy to contribute to the social dimensions in which it is immersed. It is hypothesized that the university community has different perceptions of the commitment to regional development and also, that the elaboration of the model should preferably be comprehensive and bear in mind the complexities of the regional context. The objective is to coconstruct a comprehensive regional development model of Universidad de Ibagué since the conception that agents have, from the qualitative approach and the methodology based upon the theories anchored in specific contexts of experience.

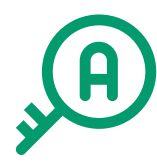

Palabras claves: Desarrollo regional, concepción de desarrollo regional, modelo de desarrollo regional integral, misión de la Universidad, formación en desarrollo regional.

Keywords: Regional development, conception of regional development, integral regional development model, mission of the University, regional development training.

\section{Introducción}

La Universidad de Ibagué es una institución de educación superior que apunta al desarrollo regional como uno de sus ejes fundamentales. Está inscrita en un territorio particular en donde quiere hacer presencia con la formación de líderes que estén comprometidos con las particularidades propias de la región. Dicha 'misión' está definida 
en los criterios y principios básicos que orientan su acción y determinan las políticas y estrategias de su funcionamiento como institución educativa. Por ejemplo, existe un semestre denominado Paz y Región, en el que los estudiantes de último semestre realizan sus prácticas profesionales en un municipio del Departamento dentro de un equipo de trabajo, en colaboración con las instituciones propias del territorio, para que de alguna manera la Universidad establezca una presencia concreta en las dinámicas y procesos de desarrollo propios de las regiones. Con este tipo de iniciativas, la Universidad manifiesta su compromiso con el desarrollo regional enfocado en los futuros líderes y empresarios que han de recibir una impronta particular que los distinga como egresados de la misma. Dicha impronta tiene que ver con el compromiso y apropiación del desarrollo regional como uno de los ejes principales de la formación personal y profesional.

Figura 1. Programa Paz y Región en Planadas (Tolima)

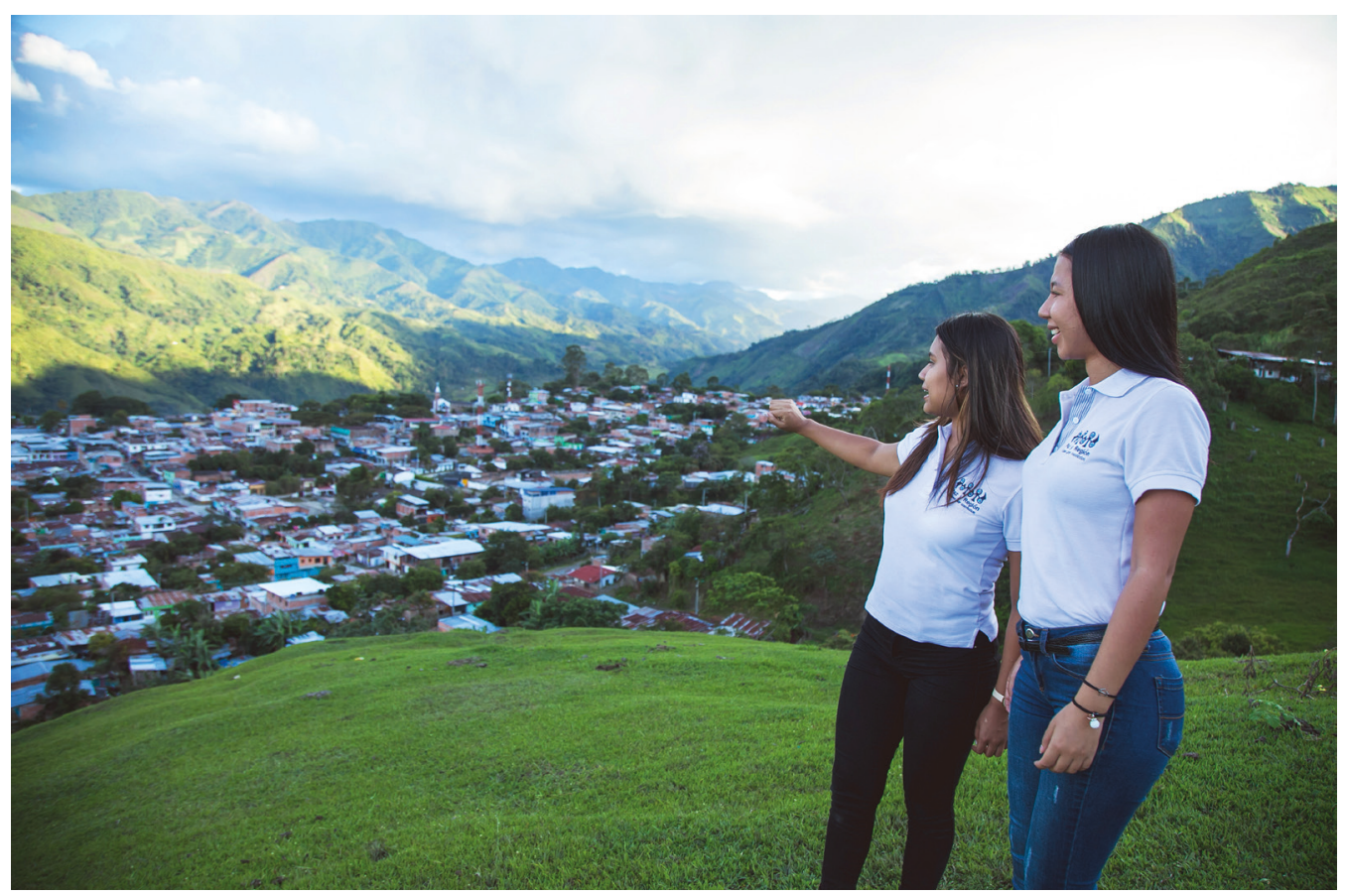

Fuente: Archivo fotográfico institucional. Comunicación Institucional Universidad de Ibagué 
No obstante, esta noción de desarrollo regional centrada en los líderes y empresarios del futuro, es decir, en los egresados de los diferentes programas de la Universidad, puedellevar a pensar el desarrollo como algo que atañe únicamente al proceso de formación de estos profesionales. En realidad, el desarrollo regional es una propiedad que se extiende sobre muchos otros aspectos que comprometen el funcionamiento de la Universidad como institución educativa. Un caso particular de esto, se vincula con la relación que tiene la Universidad con la comunidad dentro de la cual se halla inmersa. De alguna manera, la Universidad afecta el entorno concreto en el cual está inscrita y hace parte de su misión promover el desarrollo de la región.

Para que los agentes de la Universidad se puedan apropiar del desarrollo regional, se hace indispensable esclarecer los criterios propios de este, que movilicen las políticas y las estrategias de acción acordes a los retos y características propias del contexto regional. La Universidad de Ibagué tiene un modelo de desarrollo regional que no parece estar orientado desde una perspectiva integral, sino como algo fragmentado. En esta investigación trataremos de esclarecer qué es dicho desarrollo regional para la Universidad de Ibagué y desde ahí proponer un modelo integral.

La Universidad de Ibagué está "comprometida con el desarrollo regional" y debe hacerle honor al lema, el cual hace parte de su misión y visión. Pero para ello debe tener un modelo integral que impulse la tarea de cumplir con este compromiso. El propósito es estudiar ese compromiso, una vez identificadas las estrategias y actividades que lo promueven, y ver cómo se incluyen dentro de un modelo integral. Una consecuencia de este estudio, puede ser la respuesta a si es pertinente reexaminar ese compromiso, lo cual se puede definir al revisar las políticas y estrategias de acción de la Universidad, para ver si contribuyen o no al desarrollo regional, y caracterizar una concepción de este. Por lo tanto, se requiere de una investigación que ayude a construir una noción de desarrollo regional anclada desde las políticas de la Universidad y que esté articulada con el contexto particular de la región.

\section{Materiales y métodos}

\subsection{Tipo de investigación}

Esta propuesta de investigación reconoce que la coconstrucción de un modelo de desarrollo regional integral de la Universidad de Ibagué, requiere comprender la complejidad de la realidad, que es una construcción social, y entenderla como un sistema de interrelaciones. En este caso específico, la realidad es construida por la organización humana Universidad de Ibagué. Una vez se concibió este reconocimiento, se asume que la coconstrucción es un proceso colectivo en el que se dialogan las distintas perspectivas o formas de ver la situación. 
Estainvestigación seabordadesde un enfoquecualitativoutilizandolametodología de investigación acción participativa, se aplica el método de teorías fundamentadas en los contextos específicos de experiencia (Grounded Theories) según Strauss y Corbin (2002). Esta metodología permite la recolección de datos cualitativos de forma sistemática por medio de entrevistas, encuestas, observaciones, documentos, videos, películas, etc., para luego ser codificados y analizados en un proceso no matemático de interpretación, con el objetivo de descubrir conceptos y relaciones en los datos brutos y luego organizarlos en un esquema explicativo teórico, la teoría fundamentada es resultado del análisis de estos datos en un proceso de investigación (Strauss \& Corbin, 2002).

$\mathrm{Al}$ tener en cuenta lo anterior, nuestro propósito es alcanzar la concepción a partir de los datos obtenidos en el análisis del Plan de Desarrollo Institucional (PDI), Plan Educativo Institucional (PEI), informes anuales de gestión y la elaboración de entrevistas y encuestas para recoger la información. Esta muestra las concepciones de desarrollo de los distintos agentes que conforman la Universidad: estudiantes, docentes y administrativos. De esta forma, se puede identificar qué están entendiendo estos agentes y cuál es su percepción sobre el desarrollo regional. Como resultado de esta caracterización, se elabora un primer concepto desde la Universidad de Ibagué de desarrollo regional que está siendo compartido en la comunidad actual. De igual forma, también hicieron parte del proceso estudiantes de la Universidad de Ibagué como asistentes de investigación en la modalidad de monografía de grado, para contribuir al logro de los objetivos.

\subsection{Técnicas e instrumentos de recolección de información}

La revisión de las estrategias de acción, actividades y políticas se efectuará por medio de una revisión detallada de las fuentes bibliográficas, informes de gestión y documentos oficiales de la Universidad.

La recolección de datos de la concepción de desarrollo regional de los agentes se realizará por medio de encuestas con preguntas abiertas y cerradas, entrevistas y 5 grupos focales en los cuales cada agente puede describir su concepción y las actividades relacionadas a ella, de igual forma cómo se interrelacionan con las de los demás agentes. 
Figura 2. Etapa de recolección de datos

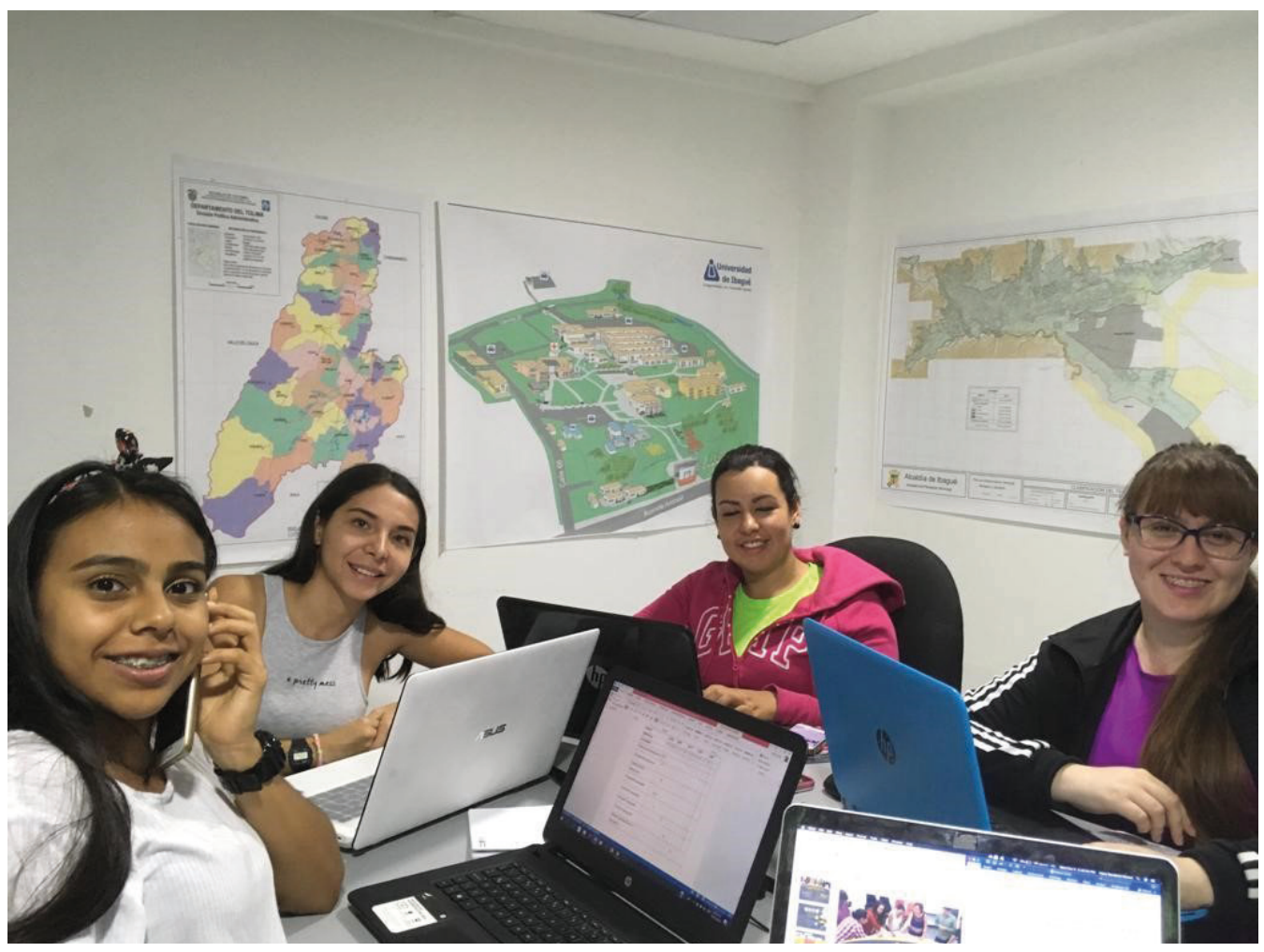

Fuente: Autores

Para el análisis comparativo constante y la categorización de la concepción, se utilizará el software NVivo. Por consiguiente, se diseñarán nubes de palabras y mapas jerárquicos que permiten observar la relevancia de las categorías que construyen la concepción de desarrollo regional en los agentes de la Universidad.

Para la coconstrucción y el codiseño del modelo de desarrollo regional integral, se propone aplicar los principios filosóficos de Design Thinking bajo la metodología diseño centrado en las personas, HCD: Human Center Desing (IDEO, 2015), su técnica de creatividad y sus herramientas, favorece los procesos de praxis (reflexión en la práctica) de la investigación acción participativa en la cocreación del producto propuesto desde y con los agentes involucrados. Esta praxis se adelanta en 4 talleres de codiseño.

Para todas las técnicas, formas de recolección de información y datos para analizar, anteriormente mencionadas, se efectuará un registro riguroso, detallado y sistemático, análisis reflexivo y comparativo constante de fotografías, notas, registros 
gráficos y audios. Este proceso permitirá la categorización de la información, la cual se presentará en nubes de palabras y pinturas enriquecidas o rich pictures. Las pinturas enriquecidas de la metodología de sistemas blandos (MSB) referida directamente de Checkland (1981), es un lenguaje visual con el que se pretende mostrar la idea o categoría central de la teoría fundada en datos, que domina a las demás, la cual hilvana y le da sentido a toda la historia que narra la pintura.

\subsection{Unidades $y$ datos}

La muestra para esta investigación será obtenida por saturación; por lo tanto, la cantidad de agentes participantes está sujeta a la profundidad y suficiencia en cada uno de los procesos de recolección de datos e información de forma participativa. La cantidad podría aumentar en la medida en que lo requieran las actividades para lograr los objetivos específicos.

Los agentes participantes serán todos aquellos que están o podrían estar involucrados en el diseño, la práctica y promoción del desarrollo regional en la Universidad de Ibagué: estudiantes, docentes y administrativos. También se considera: las directivas, Consejo de Fundadores y decanos, como agentes relevantes, puesto que están involucrados y son o podrían afectar la situación.

\subsection{Análisis e interpretación de datos}

La presente investigación hace referencia a definir criterios de desarrollo regional que movilicen las políticas y estrategias de acción, acordes a los retos y características propias del contexto de la Universidad de Ibagué.

Al considerar que los aspectos o elementos que permiten la educación para el desarrollo regional son transversales en diferentes áreas y distintas partes del currículo, se fomenta una interdisciplinariedad que amplía la comprensión y análisis de los fenómenos naturales y humanos de forma más completa en diversas dimensiones. Así mismo, la interculturalidad congregada en la educación superior reúne distintos saberes desde los conocimientos, costumbres, vivencias y experiencias, las cuales consideran particularidades históricas en distintas regiones. Al atender a la necesidad de articular el aprendizaje al contexto cultural, socio-económico, ecológico-ambiental, le da sentido a la vida cotidiana desde la academia, para aumentar la preocupación por el bienestar común desde el cuidado de los otros (seres humanos) y lo otro (medioambiente).

Las fases del proyecto fueron articuladas a cada uno de los objetivos específicos de la siguiente forma: 
INDAGAZEE e-ISSN: 2357-5042 • Número 8 (2020) • Universidad de Ibagué • doi: https://doi.org/10.35707/indagare/810

- Fase 1. Se realizó la recolección de la información, con el fin de extraer de allí una concepción para la estructuración de un modelo a partir de los documentos y datos obtenidos en esta fase. Esta información posibilitó, a través de las teorías ancladas, generar un modelo empírico de desarrollo regional.

- Fase 2. Se adelantó la coconstrucción de una concepción de los agentes de la Universidad.

- Fase 3. Finalmente, se compararon estos dos modelos de desarrollo regional, el de la Universidad de Ibagué y el coconstruido por los agentes de la Universidad, a partir de la concepción de los mismos. Las conclusiones se realizaron por medio de una síntesis.

\section{Resultados}

De acuerdo con el desarrollo del proyecto, a partir del análisis de la información, los participantes concluyeron lo siguiente:

Los agentes reconocen la importancia de varios aspectos como la economía, la calidad de vida, los problemas sociales y ecológicos (apoya las directrices de la Unesco y la ONU). Además, en el contexto del desarrollo humano sostenible, los académicos enfatizan la necesidad de equidad en las comunidades, la recuperación de valores claves y la importancia del ser humano.

La jerarquía sobre la concepción de desarrollo regional, da importancia a (en este orden): progreso, proyectos, comunidad, región, entendiéndose como territorio o localidad, y el compromiso con la comunidad en el proyecto "construcción de la ciudad y la región". La identidad de una comunidad y la relación entre quienes no participan en el proceso de desarrollo, también juegan un papel importante.

Los participantes perciben que las estrategias diseñadas para promover desarrollo regional son: Ciclo Común Básico, Contexto y Región, Introducción al Pensamiento Sistémico, Centro de Educación Regional (CERES), Responsabilidad Social y Paz y Región, Responsabilidad y Proyección Social. Otros factores importantes incluyen la formación integral de profesionales, ciudadanos y seres humanos, la difusión del conocimiento y los vínculos con los graduados y estudiantes.

Como se muestra en este estudio, se pueden implementar diferentes enfoques y estrategias para promover la educación para el desarrollo sostenible con numerosas variaciones para múltiples sujetos. Esta declaración interpreta la educación para el desarrollo sostenible como una política institucional para garantizar que las instituciones de educación superior se gestionen con el objetivo de educar y formar a ciudadanos integrales capaces de contribuir al desarrollo sostenible (Olearte-Mejía y Ríos-Osorio, 
2015). Vale la pena señalar que los resultados revelan una preocupación por la formación individual y la relación entre este individuo y el medio ambiente, sus valores, ser una persona y una formación integral como profesional y ciudadano.

Las actividades de desarrollo regional en la interfaz de usuario parecen mostrar que la conexión con la comunidad y el medio ambiente no se comprenden completamente. Sin embargo, la preocupación de la Universidad de Ibagué por una formación científica y profesional, enraizada en principios éticos y morales, puede verse como impulsada por una posición que desafía la concepción unificadora de una relación hombre-naturaleza, en la que el papel de los humanos es visto como dueño y la naturaleza como esclava.

Al seguir a Contreras, et al. (2007) y los resultados obtenidos en la Universidad de Ibagué, creemos que el desarrollo debe reformarse para dar cuenta de las interrelaciones entre los humanos, la educación, la participación y el cuidado de sí mismo, sus compañeros y sus alrededores. Creemos que esto requiere la implementación de prácticas enfocadas en la mejora de las relaciones personales y, en consecuencia, de la relación entre un individuo y el medio ambiente.

\section{Potencial uso}

El proyecto Coconstrucción de un modelo de desarrollo regional integral de la Universidad de Ibagué, es una investigación que, al partir del objetivo misional de la Universidad, basado en el desarrollo regional, es de carácter relevante para la sociedad en la cual se encuentra inmersa. Por un lado, tiene un impacto en el desarrollo de la región, puesto que este proyecto investigativo se centra en el significativo compromiso de progreso del departamento del Tolima y la importancia de que los agentes de la Universidad de Ibagué puedan

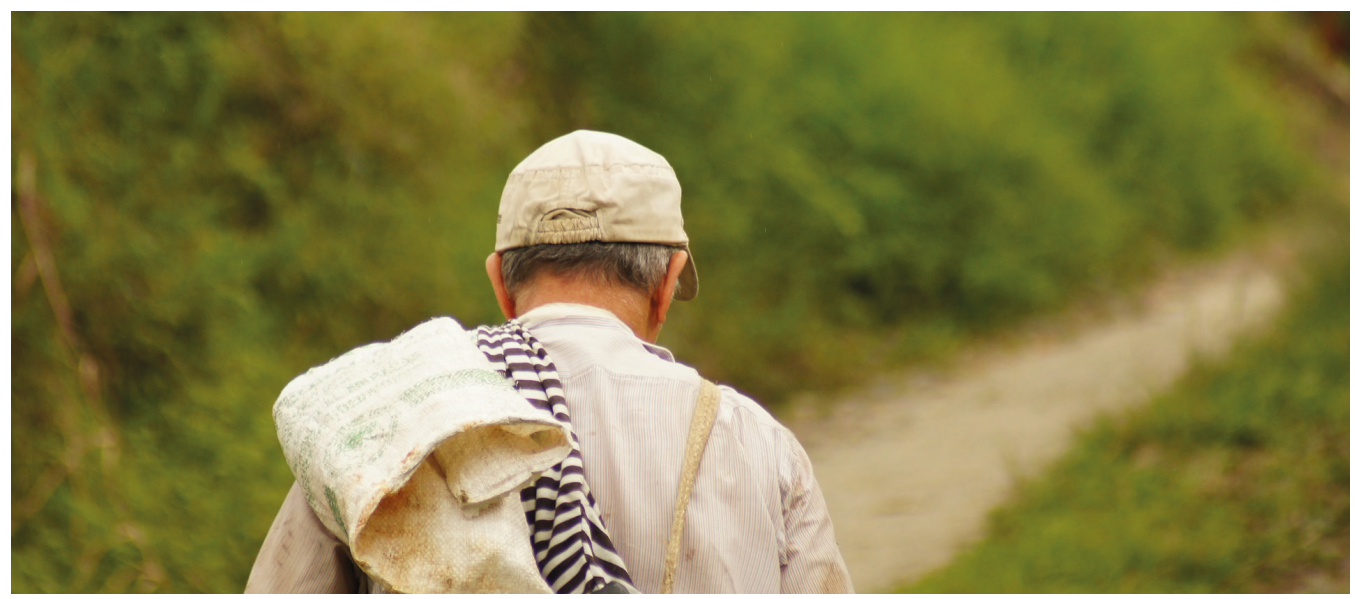


INDAGAZEE e-ISSN: 2357-5042 • Número 8 (2020) • Universidad de Ibagué • doi: https://doi.org/10.35707/indagare/810

apropiarse de este objetivo misional por medio del esclarecimiento de criterios propios, que movilicen las políticas y las estrategias de acción acordes a los retos y características propias del contexto.

Al tener en cuenta lo anterior, el establecimiento de este modelo integral tiene como propósito impulsar el compromiso de desarrollo de la Universidad de Ibagué, por medio de la identificación de estrategias eficaces que estén articuladas al contexto particular del territorio, es así como en el ámbito nacional y mundial se genera impacto, puesto que la coconstrucción de este modelo significaría un ejemplo relevante en el establecimiento de estrategias cuyo propósito sea el desarrollo de territorios nacionales y del mundo.

\section{Ficha técnica del proyecto}

Título del proyecto: Coconstrucción de un Modelo de Desarrollo Regional Integral de la Universidad de Ibagué.

PRIT: Desarrollo regional inclusivo y sustentable.

Código: 18-574-INT.

Palabras claves: Desarrollo regional, concepción de desarrollo regional.

Grupo de investigación: EULOGOS.

Investigadora principal: Lady Johanna Peñaloza Farfán.

Correo electrónico: lady.penaloza@unibague.edu.co

Coinvestigador: Hans Sanabria.

Asesor externo: Alberto Paucar-Caceres.

\section{Referencias}

Checkland, P. (1981). Systems Thinking, Systems Practice. England: John Wiley \& Sons Limited.

Contreras, J.J., Ochoa Arias, A., \& Pilonieta Blanco, C. (2007).Delfracasodeldesarrolloaldesarrolloendógeno sustentable: La nueva Organización de Desarrollo Regional. Revista Venezolana de Gerencia, 12(37). Recuperado de http://ve.scielo.org/scielo.php?script=sci_arttext\&pid=S1315-99842007000100003

IDEO (2015). Diseño centrado en las personas. Kit de herramientas. (2a Edición). Recuperado de https://drive.google.com/file/d/1y4DkMjddaPs_C6j6OlE9iKkl7QKs6LQf/view

Olearte-Mejia, D., \& Rios-Osorio, L. (2015). Enfoques y estrategias de responsabilidad social implementadas en Instituciones de Educación Superior. Una revisión sistemática de la literatura científica de los últimos 10 años. Revista de la Educación Superior, 44 (175), 19-40. https://doi.org/10.1016/j. resu.2015.10.001

Strauss, A., \& Corbin, J. (2002). Bases de la investigación cualitativa: Técnicas y procedimientos para desarrollar la teoría fundamentada. Medellín, Colombia: Editorial Universidad de Antioquia. Recuperado de https://diversidadlocal.files.wordpress.com/2012/09/bases-investigacion-cualitativa.pdf 
INDAGA3E e-ISSN: 2357-5042 • Número 8 (2020) • Universidad de Ibagué • doi: https://doi.org/10.35707/indagare/810

\section{Bibliografía recomendada}

Abidin, C., \& Reina, R. J. (2008). La Universidad como agente de desarrollo regional. Cartapacio de Derecho, 15, 1-19. Recuperado de http://www.cartapacio.edu.ar/ojs/index.php/ctp/article/view/1141

Alvarado-Borrego, A. (2009). Vinculación universidad-empresa y su contribución al desarrollo regional. $R a$ Ximhai, 5(3), 407-414. Recuperado de http://revistas.unam.mx/index.php/rxm/article/view/15171

Boisier, S. (1998). Post-scriptum sobre desarrollo regional: Modelos reales y modelos mentales. EURE, 24(72). https://doi.org/10.4067/S0250-71611998007200003

Briceño Guerrero, J. M. (1994). El laberinto de los tres minotauros. Caracas, Venezuela: Monte Ávila Editores.

De Castro Teixeira, J. B. (1997). Universidad y desarrollo regional. Archivos del presente: Revista latinoamericana de temas internacionales, 3(10), 119-123.

Fuenmayor Toro, L. (2007). La universidad venezolana y el desarrollo regional. Educere, 11(38), 489-495. http://erevistas.saber.ula.ve/index.php/educere/article/view/11507

Fuenmayor, R. (2000). Sentido y sinsentido del desarrollo. Recuperado de http://www.saber.ula.ve/ bitstream/handle/123456789/14856/sentido_y_sinsentido.pdf?sequence=1

Ministerio de Educación Nacional de Colombia (2005, agosto-septiembre). Educar para el desarrollo sostenible. Altablero. No36. Recuperado de https://www.mineducacion.gov.co/1621/article-90893. html.

Padilla Meléndez, A., \& Fuster Martín, E. (2014). Colaboración Universidad-Empresa y Desarrollo Regional. El caso de Oruro (Bolivia). Revista Venezolana de Gerencia, 19(67), 387-409. Recuperado de https://www.redalyc.org/comocitar.oa?id=29031856002

QS. (2016). QS Top Universities. (QS Quacquarelli Symonds Limited 1994 - 2016). Recuperado de QS World University Ranking: http://www.topuniversities.com/qs-world-university-rankings/methodology

Rodríguez Rodríguez, J. (2009). La gestión del desarrollo regional sostenible: Un desafío desde la Universidad Autónoma de Manizales. Ánfora, 16(26), 5-18. Recuperado de https://doi.org/10.30854/ anf.v16.n26.2009.127

Unesco (2012). Exploring Sustainable Development: A Multiple-Perspective Approach. Paris: Education for Sustainable Development Section (ED/PSD/ESD).

Unesco (2016). Educación para el Desarrollo Sostenible. Recuperado de: http://www.unesco.org/new/en/ education/themes/leading-the-international-agenda/education-for-sustainable-development/

Universidad de Ibagué (2014). Plan de Desarrollo Institucional 2014-2018: PDI. Recuperado de https://hdl. handle.net/20.500.12313/368

Universidad de los Andes (2016). Programa de Desarrollo Integral (PDI 2016-2020). Recuperado de https:// planeacion.uniandes.edu.co/pdi/nuestro-pdi

Vallejo Mejía, C. (2006). Nuestro compromiso es con el desarrollo regional. Ánfora, 13(20): 4-8. https://doi. org/10.30854/anf.v13.n20.2006.241 